\title{
Reproducción Estacional en el Macho
}

\author{
Seasonal Reproduction in the Male
}

****Eduardo Bustos Obregón \& ** Leandro Torres -Díaz

BUSTOS OBREGÓN, E. \& TORRES, L. Reproducción estacional en el macho. Int. J. Morphol., 30(4):1266-1279, 2012.

RESUMEN: Hay una sorprendente relación entre el ambiente y adaptaciones de la conducta reproductiva, muy evidente en los reproductores estacionales que pueden reproducirse en días cortos o largos, de acuerdo a factores proximales, especialmente el fotoperiodo luminoso que provoca cambios fotoneuroendocrinos. Estos, involucran fotoreceptores, un reloj biológico y el aparato neuroendocrino. Las gonadotrofinas (GT), el desarrollo gonadal, la retroalimentación negativa de las GT por los esteroides sexuales, la intervención de las fibras retino-hipotalámico y los núcleos supraquiasmáticos así como la secreción de melatonina, intervienen en esta regulación. El pulso generador del hipotálamo (eminencia media) es importante en el control de la adenohipófisis respecto de la secreción de LH y FSH. En el testículo, las endocrinocitos intersticiales (de Leydig) (que secretan testosterona y también estrógenos), establecen un asa de retroalimentación con la adenohipófisis y el hipotálamo en un circuito de asa larga, corta y ultracorta, donde neuronas neuroendocrinas tienen un rol importante. Los sustentocitos (células de Sertoli) (intratubulares) son importantes por su rol mecánico, trófico y metabólico respecto a las células germinales y la secreción de activina e inhibina, que provoca o inhibe la secreción de FSH respectivamente. Los sustentocitos también secretan muchas proteínas específicas entre las cuales se encuentra la proteína que liga andrógenos (ABP), importante porque concentra 100 veces la testosterona en el parénquima testicular. La secreción tónica, por pulsos de GT, especialmente LH, es debida a actividad hipotalámica a través del control de generación de estos pulsos que se inicia en la pubertad. La reproducción en el potro y el toro se presentan como ejemplos.

PALABRAS CLAVE: Reproducción estacional; eje Hipotálamo-Hipófisis-Testículo; Neuroendocrinología y reproducción; Características reproductivas del potro y el toro.

\section{INTRODUCCIÓN}

Las condiciones ambientales dominan el desempeño reproductivo de modo que los animales pueden ajustarse, y a su vez anticiparse, a los cambios que ocurren a su alrededor. Esto puede requerir una respuesta rápida como lo vemos en los ratones machos, los que al ingresar a un nuevo territorio secretan una feromona que induce que las hembras ingresen al estro en solo 3 días. Esto le permite poblar el ambiente rápidamente de modo de pasar una mayor cantidad de sus genes a la siguiente generación. Igualmente, la respuesta reproductiva al cambio ambiental puede tomar un tiempo mayor en animales que, dado su tamaño, tardan más tiempo en desarrollarse, como es el caso de los equinos y bovinos.

Un evento común en las especies silvestres y en algunos casos en especies de producción, es la reproducción estacional, estrategia que mejora sustancialmente los procesos reproductivos y que por definición es regulada por el ambiente. Las ventajas de generar animales juveniles solo en el período en el cual las condiciones son favorables ha sido ejemplificado en muchas especies de vida silvestre, en las que por lo general, el factor clave en determinar cuántos animales jóvenes pueden generarse es la disponibilidad de alimento que está presente en el medio en el período del nacimiento.

Por lo tanto, los corderos nacen en primavera cuando la presencia de pasto verde recién crecido puede sostener la lactancia y promover el destete de las crías.

La lista de adaptaciones específicas es interminable, pero el factor común siempre el mismo: hay un tiempo óptimo para el nacimiento de las crías en cada especie, determinado principalmente por el periodo gestacional de modo que se debe asegurar que los descendientes nazcan cuando las temperaturas ambiéntales sean más cálidas y las disponibilidad de comida incremente que es por lo general, la primavera e inicios del verano (Reiter, 1980; Reiter et al., 2009). Los preparativos para la reproducción deben comenzar con la suficiente antelación para otorgar el tiempo adecuado para

* Universidad de La Frontera, VRIP, Temuco, Chile.

**Laboratorio de Biología de la Reproducción, Programa de Anatomía y Biología del Desarrollo, ICBM, Facultad de Medicina, Universidad de Chile, Santiago de Chile. 
el desarrollo gonadal, la producción de gametos, el establecimiento de territorios de reproducción y gestación. Este período puede tomar a lo menos dos meses en roedores pequeños y hasta un año o más en mamíferos superiores. De esto se deduce que los animales son dependientes de las señales ambientales, que son a menudo denominados factores proximales, los que le informarán cuando iniciar su proceso reproductivo.

En teoría, cualquier factor ambiental puede ser usado, pero la evolución asegura que solo los indicadores más seguros son seleccionados. Sin embargo, una gran gama de factores ambientales desencadena la competencia reproductiva. Por ejemplo, las Jirafas tienen sus crías cuando los árboles de acacia tienen las hojas crecidas, y algunos animales han construido relojes anuales que miden estos ritmos de la naturaleza de manera constante y aguda de modo que se han vuelto extremadamente vitales para la sincronización de la reproducción.

Los trópicos tienen condiciones ambientales más o menos estables durante todo el año debido a que el sol recae sobre estos territorios de igual forma durante todo el año, sin embargo, mientras más nos alejamos de los trópicos la reproducción estacional se vuelve cada vez más evidente y precisa en su sincronización.

Afortunadamente, la inclinación del eje de la tierra y su órbita anual alrededor del sol provee un factor proximal ideal en forma de cambios anuales en el fotoperíodo (duración de los periodos de luz), por lo que aves y mamíferos usan generalmente la duración de la luz del día, para regular no solo su reproducción si no también muchos otros procesos estacionales como la hibernación, migración, apetito, pelaje, crecimiento, mudas, etc.

Reproductores de día corto y reproductores de día largo. Si una especie alcanza su madurez reproductiva completamente en día corto o en día largo, depende esencialmente de la duración de su gestación. Generalmente, roedores y carnívoros cuyas épocas de preñez varían entre 20 y 80 días, son reproductores de día largo y su época de apareamiento es en primavera y verano. Sin embargo, en esta categoría hay diferencias entre las especies, algunas de las cuales pueden involucrar diferencias fisiológicas considerables. El ciclo de muchos roedores pequeños, por ejemplo, termina al final del verano cuando el acortamiento del día combinado con la disminución en las reservas alimenticias y las bajas temperaturas causan la regresión de sus gónadas. En algunos animales de reproducción estacional, la gónada del macho puede disminuir su tamaño entre un 10 y un $95 \%$ en respuesta a los factores ambientales asociados a su estación no reproductiva (Young \& Nelson, 2001).
Esta inhibición fotoperiódica continúa por meses. Sin embargo, luego de un tiempo, desaparece y las gónadas crecen espontáneamente a pesar de prevalecer los días cortos, de modo que la mayoría de los roedores pueden reproducirse también a inicios de la primavera. Los días largos estimulan y mantienen a los animales en reproducción. Sin embargo, también hacen que el roedor se haga sensible a los efectos inhibitorios de los días cortos, que vendrán en otoño, donde nuevamente sus gónadas regresionan.

La explicación de este fenómeno ha sido esclarecida a partir de los experimentos realizados en el Hamster Dorado Mesocricetus aureatus y en Hamster Djunjarian, Phodopus sungorus, pero es probablemente aplicable a la mayoría de los roedores pequeños y animales de producción, aunque los detalles varían dependiendo de las situaciones particulares de cada especie ya que el grado de control fotoperiódico depende de las latitudes del planeta en la que se encuentren las poblaciones. Un ejemplo lo constituyen los felinos silvestres de Centroamérica, en cuanto a la latitud y la duración de la luz del día. Desde el Ecuador a $20^{\circ} \mathrm{N}$, los gatos salvajes se reproducen en cualquier momento del año, pero en la medida en que las latitudes de incrementan, se vuelven gradualmente estacionales, de hecho, entre las latitudes $50-60^{\circ} \mathrm{N}$ se producen camadas solo entre Marzo y Septiembre. En el laboratorio, se ha replicado este fenómeno y frente a regímenes de luz 12:12 (L:O; Luz:Oscuridad) como ocurre en el Ecuador, los felinos se reproducen continuamente con un porcentaje del $4 \%$ de cruzas por semana; sin embargo, si son sometidos a regímenes de días cortos de 9:15 (L:O) se reduce el rango al 1\% por semana, mientras que bajo regímenes 14:10 (L:O) se incrementa a un $10 \%$.

En mamíferos superiores como las ovejas, cabras y ciervos, las gestaciones son largas, entre 148 y 250 días, por lo que su reproducción tiene lugar durante los días más cortos en el otoño. Este efecto fotoperiódico puede ser inducido artificialmente si las ovejas Suffolk son sometidas a 8:16 (L:O), lo que hace que éstas ingresen a la fase de Estro, luego de dos meses cuando se expondrán al rango de luz día normal al aire libre. Siempre que la preñez no ocurra, las ovejas seguirán teniendo ciclos estrales de entre 16-17 días. Sin embargo, la época de apareamiento puede terminar prematuramente por exposición de las ovejas a días largos.

Si en los animales el largo de la preñez se aproxima a un año, sus temporadas de apareamiento corresponden al período de primavera-verano. El equino (cuya gestación dura 11 meses), pertenece a esta categoría. Las yeguas por ejemplo, presentan un anestro de invierno y ovulan solo en el período comprendido entre el verano y el otoño. La selección artificial asociada a una excelente dieta, pueden redu- 
cir la proporción de hembras que entran en anestro en invierno, pero la fertilidad en invierno es muy inferior. La exposición de las hembras a fotoperíodos largos genera una mayor producción de animales. Por otro lado en el potro, el diámetro de los túbulos seminíferos es mayor en el período reproductivo que en otros meses del año. Además, la concentración de testosterona dentro del parénquima testicular ha mostrado ser dos veces mayor durante primavera y verano que en el invierno, donde íncluso se ha asociado un pico de concentración de testosterona con rangos de producción de espermatozoides $65 \%$ superiores que los observados en invierno (Berndtson et al.,1983) .

\section{CONTROL FOTOPERIODICO DE LA REPRODUC- CION.}

La Maquinaria Fotoneuroendocrina. En términos simples, el fotoperíodo requiere de 3 componentes esenciales para realizar su trabajo. Primero, debe haber un fotorreceptor que detecte la luz y un reloj que distinga días largos de días cortos. Segundo, debe haber una ruta neural que enlace dicho reloj al aparato neuroendocrino. Finalmente, se encuentra el propio sistema endocrino, que involucra a la secreción de gonadotrofinas hipofisiarias, el desarrollo gonadal y la retroalimentación gonadal producida por los esteroides sexuales. Se sabe que hay componentes que pueden modificarse o sustituirse dependiendo de los factores ambientales (luz, alimento, etc.) que sean utilizados como indicador de condiciones favorables para la reproducción (Fig. 1). Sin embargo, en la práctica ha sido posible investigar esos componentes solo de manera relativa en algunas especies en el laboratorio como: el hamster, el gorrión, la oveja y más recientemente en el potro. Actualmente, se ha logrado esclarecer el control neurológico e incluso molecular que ejerce la luz sobre la capacidad reproductiva del organismo.

Fotorrecepción y Reloj Biológico. Los mamíferos usan sus ojos como fotorreceptores para medir el largo del día, pero la ruta por la que lo hacen es diferente a la de la captación y formación de imágenes. La información fluye a lo largo del nervio óptico (NO) en neuronas diferentes a las del sistema visual, las que son precisamente células ganglionares que contienen un único fotopigmento denominado Melanoxina (Panda et al., 2005;). Estas terminan en los núcleos supraquiasmáticos, dos estructuras discretas localizadas en el hipotálamo justo sobre el quiasma óptico (Moore et al., 1995; Hattar et al., 2002) las que en conjunto constituyen el tracto retino-hipotalámico (Fig. 2).

El dispositivo que mide el tiempo, parece estar basado en un reloj circadiano (de aproximadamente 24 horas) localizado en el núcleo supraquiasmático. Cada una de estas pequeñas áreas contiene algunos miles de neuronas, que

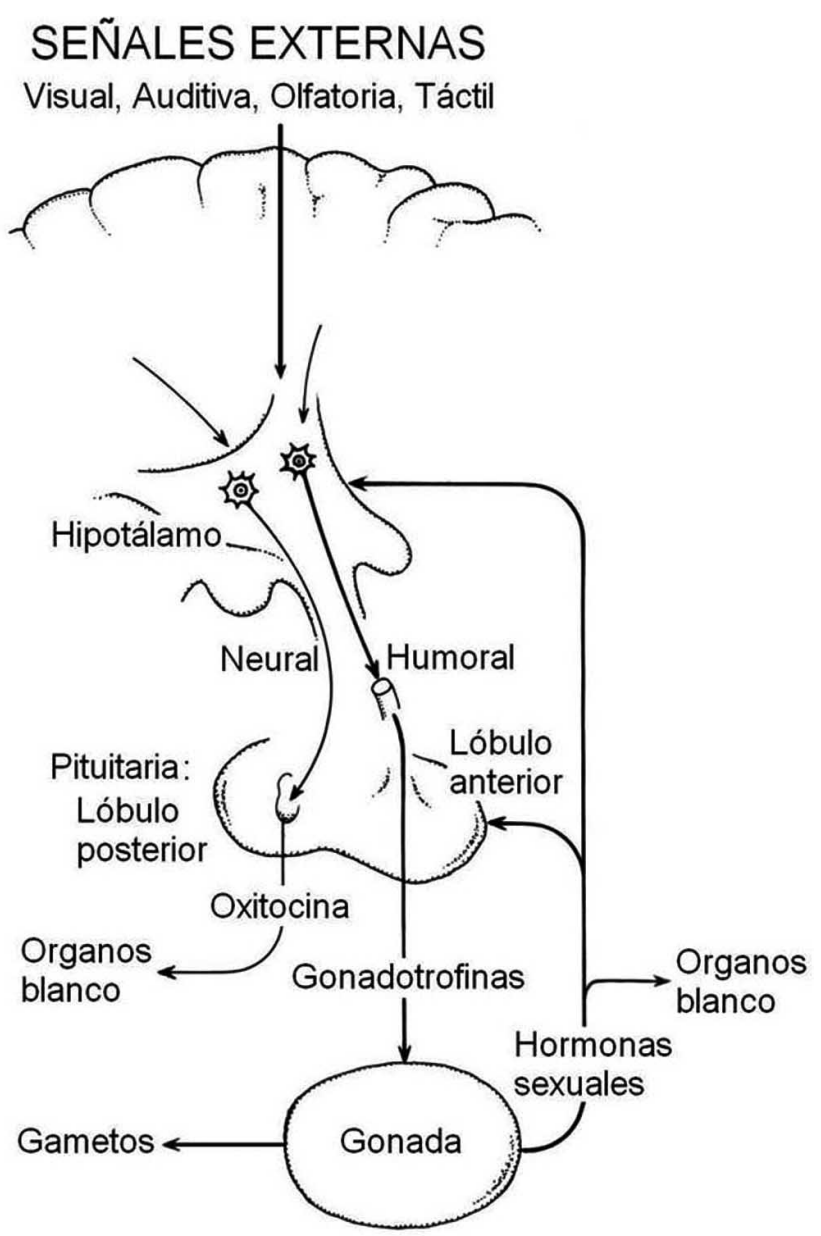

Fig. 1. Señales externas que intervienen en la producción gamética en animales de reproducción estacional y su relación con la actividad gonadal.

regulan todos los ritmos circadianos del cuerpo, por lo que su destrucción desprograma los ritmos alternantes entre sueño y vigilia, la función adrenal y el control de la temperatura corporal. Este reloj fotoperiódico también ha mostrado cesar sus funciones después de lesiones, como en el Hamster por ejemplo, donde se simula una situación de día largo permanente imposibilitando la regresión de sus gónadas bajo días cortos (Reiter, 1973).

Una predicción que nace del concepto del reloj circadiano es que la inducción de la reproducción depende de la temporalidad de los eventos lumínicos y no de su permanencia. En base a esto se han realizado diseños experimentales en los cuales la aplicación de dos pulsos cortos de luz, uno que actúa como "amanecer" y otro que actúa como "anochecer" y la variación en la cercanía entre ambos pulsos, determina cómo el animal en diferentes grados de lejanía temporal, puede leer el período como si fuese un día largo o corto. Este experimento ha sido realizado en un gran 


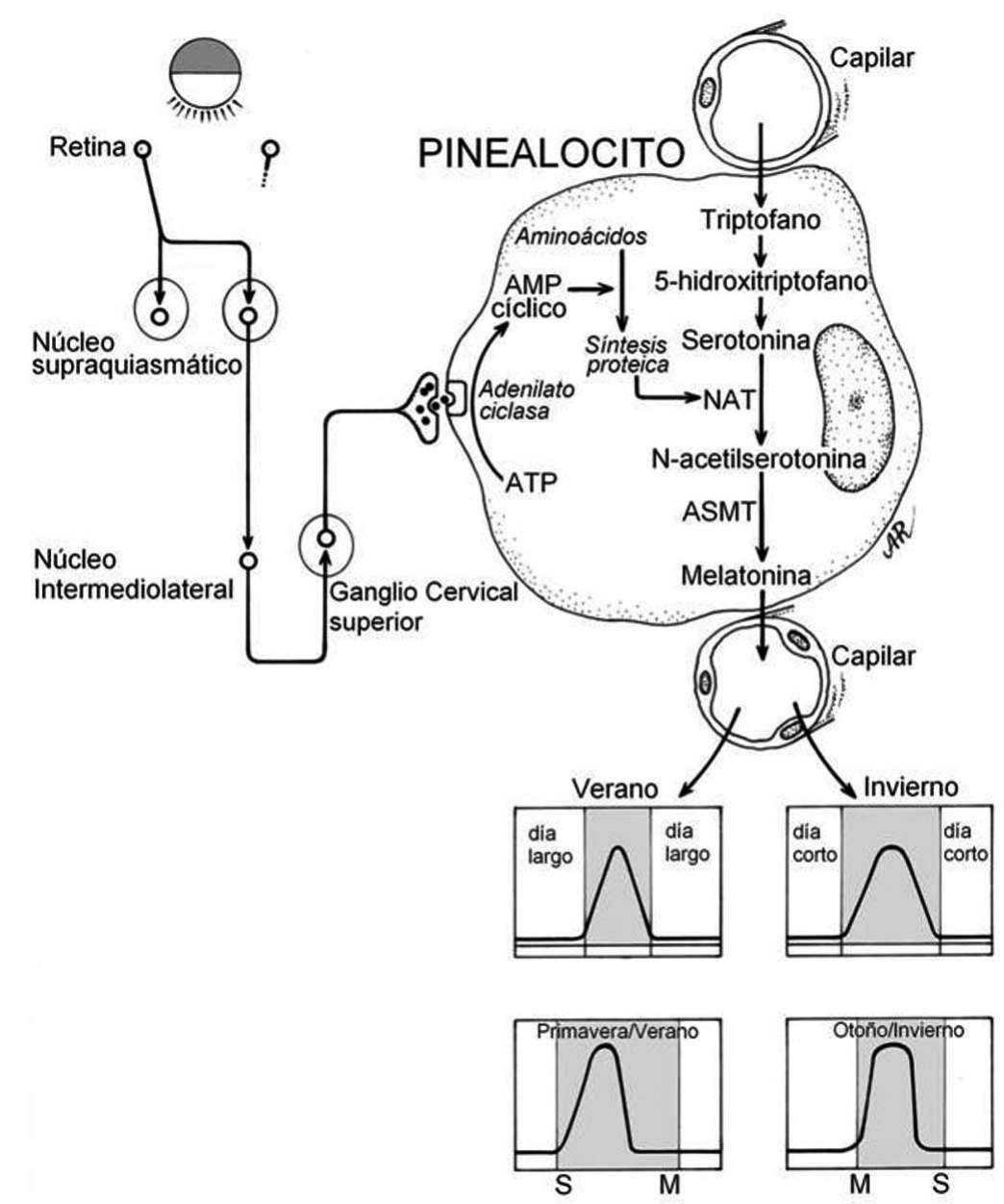

Fig. 2. Esquema del tracto fotoneuroendocrino y modificaciones el la sintesis y secreción de melatonina en reproductores de día largo (verano) y reproductores de día corto (invierno).

número de especies. Por ejemplo, si el segundo pulso de luz ocurre entre 11 a $15 \mathrm{hrs}$ después del primer pulso de "amanecer", las codornices actúan como si el día fuese largo y sus gónadas se desarrollan como lo harían normalmente. Cualquier otra combinación es interpretada como un día corto. Estudios posteriores han documentado que la duración absoluta del aumento de la melatonina nocturna es el mayor determinante en la inducción tanto de la actividad como de la inactividad reproductiva (Elliott, 1976; Carter et al., 1983) (Fig. 1).

Enlace Neural-Endocrino. En reproductores no estacionales, la información que fluye desde los somas de las neuronas GnRH en el hipotálamo anterior regula la secreción de gonadotrofinas por la hipófisis anterior, sin la intervención de otros elementos. Una ruta un poco diferente se expresa en mamíferos de reproducción estacional, donde la glándula pineal actúa como el principal transductor neuroendocrino.

La principal fuente de información sobre este fenómeno ha sido aportada por algunos grupos de laboratorio realizando estudios en el Hamster. Sin embargo, trabajos similares se han realizado tanto en la oveja como en otras especies. Luego de que la información lumínica ha sido procesada en el núcleo supraquiasmático, los impulsos nerviosos atraviesan el tronco cerebral y bajan hacia la médula espinal. Los nervios simpáticos que salen de la región torácica de la médula, ascienden hacia el cuello y hacen sinapsis en los ganglios cervicales superiores (Kappers, 1976). Desde ahí los nervios simpáticos post ganglionares viajan a la cabeza e inervan muchas estructuras incluyendo la glándula pineal. La liberación de noradrenalina a los pinealocitos por parte de las neuronas simpáticas al inicio de cada noche regula la elevación en la producción y liberación de melatonina a la circulación nerviosa y sistémica (Reiter et al., 1991). Normalmente esto ocurre solo en la noche, donde la actividad de la glándula pineal es regulada por el reloj circadiano presente en el núcleo supraquiasmático. En algunos animales las inyecciones nocturnas de melatonina pueden causar regresión testicular.

En el laboratorio se han tratado machos con infusiones de melatonina en hamster pinealoectomizados por 4 a 6 horas diarias imitando el patrón de secreción de día largo o por 10 a 12 horas como ocurre en días cortos. La regresión testicular ocurre solo en el segundo caso. El animal por consiguiente posee un calendario en la forma de un perfil de secreción de melatonina, el que es interpretado de manera apropiada por el hipotálomo. La palabra "apropiado" es usada debido a que la glándula pineal no ha sido asociada solo a la reproducción estacional sino también en otros cambios fotoperiódicos. Entre estos cambios se incluye el incremento en la secreción de Prolactina, aumentos en el consumo de alimento en los días largos del verano y cambios en el crecimiento y color del pelaje en el hamster, visones y ovejas, en los días cortos. Otro ejemplo de este fenómeno se ha demostrado mediante la destrucción de la inervación simpática que regula la actividad de la glándula pineal (por ejemplo, por glanglioectomía cervical superior bilateral) con lo que la producción de Melatonina nocturna no ocurre y la glándula no regula la fisiología reproductiva (Reiter \& Hester, 1966). De este modo los animales sometidos a dicha manipulación se vuelven 
reproductores continuos, lo que generaría una situación problemática para animales de vida silvestre que habitan en zonas donde las condiciones ambientales varían de manera cíclica.

Debido a la regresión de las gónadas asociada a un aumento de la melatonina por periodos prolongados se han aplicado a ésta los términos de "antigonadal" y "antigonadotrófico". Sin embargo, hoy se sabe que muchos reproductores de día corto como ovejas y ciervos, son sexualmente más activos y capaces de reproducirse durante los días más cortos del año, cuando los niveles de melatonina son máximos en términos de su secreción nocturna (Coelho et al., 2006; Wagner et al., 2008; Chemineau et al., 2008). En estas especies entonces, podemos decir, que melatonina actúa como factor que regula positivamente la secreción de gonadotrófinas (Reiter et al.)

Obviamente la melatonina no es un antigonadotrófico en sí mismo ni tampoco un pro-gonadotrífico, sino más bien, la duración de su secreción nocturna actúa como un mensaje pasivo que provee información al eje hipotálamo-hipófisis-gónada (información calendaria) (Reiter, 1993) activando o desactivando su acción.

Los mecanismos celulares y moleculares por los cuales melatonina interviene a nivel del eje neuroendocrino en la modulación de la secreción de gonadotrofinas y prolactina se empiezan a conocer (Morgan et al., 2008), incluyendo los receptores celulares que median los efectos de melatonina (Reppert, 1997; Duncan et al., 2007; Revel et al., 2007) con lo que se están comprendiendo algunos de los complejos mecanismos por los cuáles melatonina actúa como un agente modulador de la función reproductiva.

Como se sabe que la acción de melatonina en reproductores de día corto facilita su reproducción se ha propuesto la utilización de ésta como un agente farmacológico que anticipa la estación reproductiva en la oveja en la que ha mostrado inducir los ciclos estrales e incrementar el número de partos en intervalos donde normalmente éstas presentan un anestro estacional (Haresign et al., 1992; Abecia et al., 2007).

El sistema endocrino: secreción de gonadotrofinas. La respuesta primaria a la fotoestimulación es el incremento en la secreción de LH y FSH por la hipófisis. Esto es obviamente observable en los machos, donde los testículos crecen enormemente en algunas semanas. La secreción de FSH se incrementa cerca de 40 veces 10 días después del cambio de fotoperíodo en algunos animales y es fácilmente medible en una muestra de sangre. Los espermatozoides aparecen después de un mes en el hamster, el que es completamente fértil luego de seis semanas. Un patrón similar ha sido observado en carneros, ratas y hombres. Se ha sugerido que las escalas de tiempo en la que ocurren estos sucesos son diferentes en las especies. Sin embargo la endocrinología del desarrollo gonadal es común en todos: lo que difiere es el mecanismo neurológico usado para activar el complejo hipotálamo-hipófisis.

Un análisis temporal progresivo de la secreción de FSH y LH genera una imagen con una marcada regularidad en las oscilaciones de LH. A partir de estas observaciones se ha desarrollado la idea de que el hipotálamo contiene un "generador de pulsos" que se desencadenan a causa de una descarga coordinada de GnRH desde la eminencia media hipotalámica. La mejor evidencia de este hecho ha sido observada en la oveja, en la cual el número de episodios secretorios de LH aumenta de dos a tres veces entre verano e invierno.

La GnRH es liberada a la circulación portal hipofisiaria, llega hasta los gonadotropos (células productoras de gonadotrofinas) presentes en la hipófisis anterior (adenohipófisis) donde induce la secreción de FSH y LH. Luego de su liberación, éstas viajan por la circulación sistémica hasta los testículos, donde FSH ejerce sus efectos en los sustentocitos (células de Sertoli) mientras que LH lo hace sobre los endocrinocitos intersticiales (células de Leydig). Los sustentocitosse localizan al interior de los túbulos seminíferos del testículo y su función principal es apoyar el desarrollo de los espermatozoides. Los sustentocitos secretan además algunas proteínas que regulan la liberación de FSH desde la hipófisis anterior (inhibina y activina) y proteínas de unión a testosterona (ABP; Androgen Binding Protein). Los endocrinocitos intersticiales se localizan entre los túbulos seminíferos, y por la estimulación de LH inician la síntesis y secreción de testosterona. Además, estas células secretan en los potros altas concentraciones de estrógenos. Sin embargo, su función fisiológica exacta no se conoce.

La testosterona es necesaria para el comportamiento sexual normal, la función testicular y el desarrollo muscular.

Las variaciones estacionales en las concentraciones de FSH, LH y testosterona, han sido relacionadas con variaciones en el número de sustentocitos en el potro en la época reproductiva (Johnson \& Thompson, 1983).

De hecho, las concentraciones testiculares de testosterona son considerablemente superiores a las concentraciones sistémicas, y son necesarias para la función normal. Las proteínas producidas por los sustentocitos que se unen a la testosterona son las responsables de la mantención de esta alta concentración testicular. 
Los niveles de testosterona controlan tanto la liberación de GnRH como de gonadotrofinas (y por ende su propia concentración) a través de un sistema de retroalimentación negativa.

Cuando las concentraciones de testosterona son altas, este sistema disminuye la producción de ésta debido a la inhibición que se produce sobre el hipotálamo y la hipófisis. Por el contrario, cuando las concentraciones de testosterona son bajas, no ocurre tal inhibición y el sistema incrementa la producción de testosterona.

El control hormonal se encuentra bajo una modificación estacional. En períodos de día corto, la liberación de GnRH es inhibida por melatonina, lo que se traduce en una disminución en la liberación de LH y la subsecuente baja secreción de testosterona. Así, durante los períodos de día corto (en el invierno), la función reproductiva del macho es suprimida debido a las bajas concentraciones de testosterona. Durante este período el tamaño testicular y la producción de espermatozoides se reducen, y el comportamiento sexual varía, y no es poco común observar un comportamiento sexual disminuido (líbido), y así el animal requiere un tiempo mayor para la monta y eyaculación. Los potros pueden ser puestos bajo regímenes de luz artificial con el fin de incrementar la actividad testicular por lo que son expuestos a días largos de 16 horas al inicio de Diciembre.

El tamaño testicular y la producción de espermatozoides se aproximan a los valores normales de la época reproductiva aproximadamente luego de 60 días postexposición.

En el carnero, el tamaño de los testículos se relaciona con las concentraciones de FSH, LH y testosterona en la sangre, donde se ha observado una relación lineal entre el tamaño testicular y los niveles de FSH, también se ha observado una correlación positiva entre el número de espermatogonias en renovación y los valores medios de $\mathrm{LH}$ en la sangre periférica en el adulto (Courot \& Ortavant, 1981).

\section{EJE HIPOTALAMO-HIPOFISIS- TESTICULO}

Hipotálamo y adenohipófisis. El cerebro puede dirigir la actividad de la hipófisis anterior aun cuando actualmente queda por definir aspectos importantes de ésta regulación. Se sabe que información visual, auditiva, táctil y olfatoria registrada por el sistema nervioso central converge hacia el hipotálamo y allí es procesada, amplificada y transducida a señales hormonales que se transmiten a la adenohipófisis donde nuevamente es amplificada y retransmitida a través de la gonadotrofinas hacia las gónadas, las cuales respon- den de varios modos, uno de los cuales es secretando las hormonas sexuales. Estas últimas actúan en tejidos blancos que incluyen al propio cerebro y la adenohipófisis, así se constituye una red de información que permite la propagación e integración de señales en el organismo.

Hipotálamo. El hipotálamo forma la base del cerebro. Está limitado frontalmente por el quiasma óptico, caudalmente por los cuerpos mamilares, y dorsalmente por el tálamo. El hipotálamo rodea al tercer ventrículo y comprende grupos de neuronas con sus axones y terminales, axones y terminales de otras neuronas cuyos cuerpos están fuera del hipotálamo, y axones que pasan por este órgano. También posee células gliales como estructuras de soporte y un tipo especial de circulación (portal) en la región de la eminencia media. Las neuronas hipotalámicas se agrupan en núcleos, y envían proyecciones axonales a alguna de cuatro regiones generales:

1. Otras áreas del cerebro

2. Otros núcleos hipotalámicos

3. Eminencia media

4. El lóbulo posterior de la hipófisis

Así se constituye una compleja red de comunicaciones neuronales e interacciones entre centros regulatorios nerviosos y endocrinos.

La eminencia media es un área de confluencia de mensajes nerviosos y sanguíneos que regula la función de la adenohipófisis. Comprende la base del hipotálamo y se continúa con el tronco infundibular. Consiste principalmente de axones y terminales de neuronas hipotalámicas y extrahipotalámicas, células de la glia y células ependimarias (tanacitos). Éstas últimas tapizan el tercer ventrículo y transfieren información del líquido cerebroespinal a la adenohipófisis. La eminencia media contiene además plexos capilares conectados con el sistema porta hipotálamohipófisis. Debido a ésta anatomía, el hipotálamo tiene especial rol como interfase entre el sistema nervioso central y el sistema endocrino.

Hipófisis. La glándula consiste de lóbulo anterior (adenohipófisis), y posterior (neurohipófisis) subdividido en infundíbulo y tronco infundibular.

El proceso infundibular contiene neuronas cuyos cuerpos residen en el hipotálamo, estableciendo contacto directo entre el lóbulo posterior y el cerebro, conexión de interés para la secreción de hormonas desde la hipófisis posterior. El lóbulo anterior se subdivide en parte distal, parte intermedia, y parte tuberal. La parte tuberal rodea al infundíbulo como una manga y se extiende hacia arriba a 
la región de la eminencia media. La hipófisis anterior no contiene fibras nerviosas ni terminales. Está conectada con el cerebro por conexiones vasculares, el sistema portahipotálamo-hipófisis. Éste tiene plexos capilares en la eminencia media, con vasos que cursan por el tronco infundibular para terminar en plexos capilares secundarios en la adenohipófisis. La hipófisis anterior consiste en distintos tipos celulares con características citológicas y tintoriales distintas. Hay células especializadas en la síntesis y secreción de cada una de las 6 hormonas de la hipófisis anterior a excepción de las gonadotrofinas LH y FSH. Se acepta que el mismo tipo celular (gonadótropo) secreta a ambas. El otro tipo celular importante en reproducción son los lactótrofos que secretan prolactina. Las otras hormonas tróficas no tienen rol importante directo en la reproducción.

LH y FSH son glicoproteínas constituidas por 2 cadenas peptídidas (subunidades alfa y beta). La cadena alfa de ambas es idéntica pero la beta es distinta y le confiere su especificidad biológica. Prolactina en cambio consiste en una sola cadena peptídica.

\section{LA ORGANIZACIÓN FUNCIONAL DEL EJE HIPOTÁLAMO-HIPÓFISIS (FIG. 3 y 4)}

Neuronas endocrinas. La mayoría de las neuronas descargan sustancias neurotransmisoras de sus terminales en una sinapsis modulando así la actividad de neuronas postsinápticas. Muchas neuronas hipotalámicas son diferentes ya que no liberan neurotransmisores de su terminal a una sinapsis sino liberan hormonas al torrente sanguíneo. Así estas hormonas modulan funciones de células a distancia. Las neuronas endocrinas tienen como toda neurona cuerpo, dendritas y axón y son capaces de generar potenciales de acción. Su actividad es influida por neuronas tradicionales. Hay 2 tipos de ellas en el hipotálamo: neuronas magnocelulares y parvocelulares. Sus cuerpos se ubican en los núcleos supraóptico y paraventricular y sus axones viajan por el hipotálamo a la eminencia media y luego al tronco infundibular para terminar en la neurohipófisis. Las neuronas magnocelulares sintetizan y secretan hormonas de la neurohipófisis (oxitocina y vasopresina). Las neuronas parvocelulares tienen sus cuerpos en diversos núcleos hipotalámicos y sus axones terminan en la eminencia media.

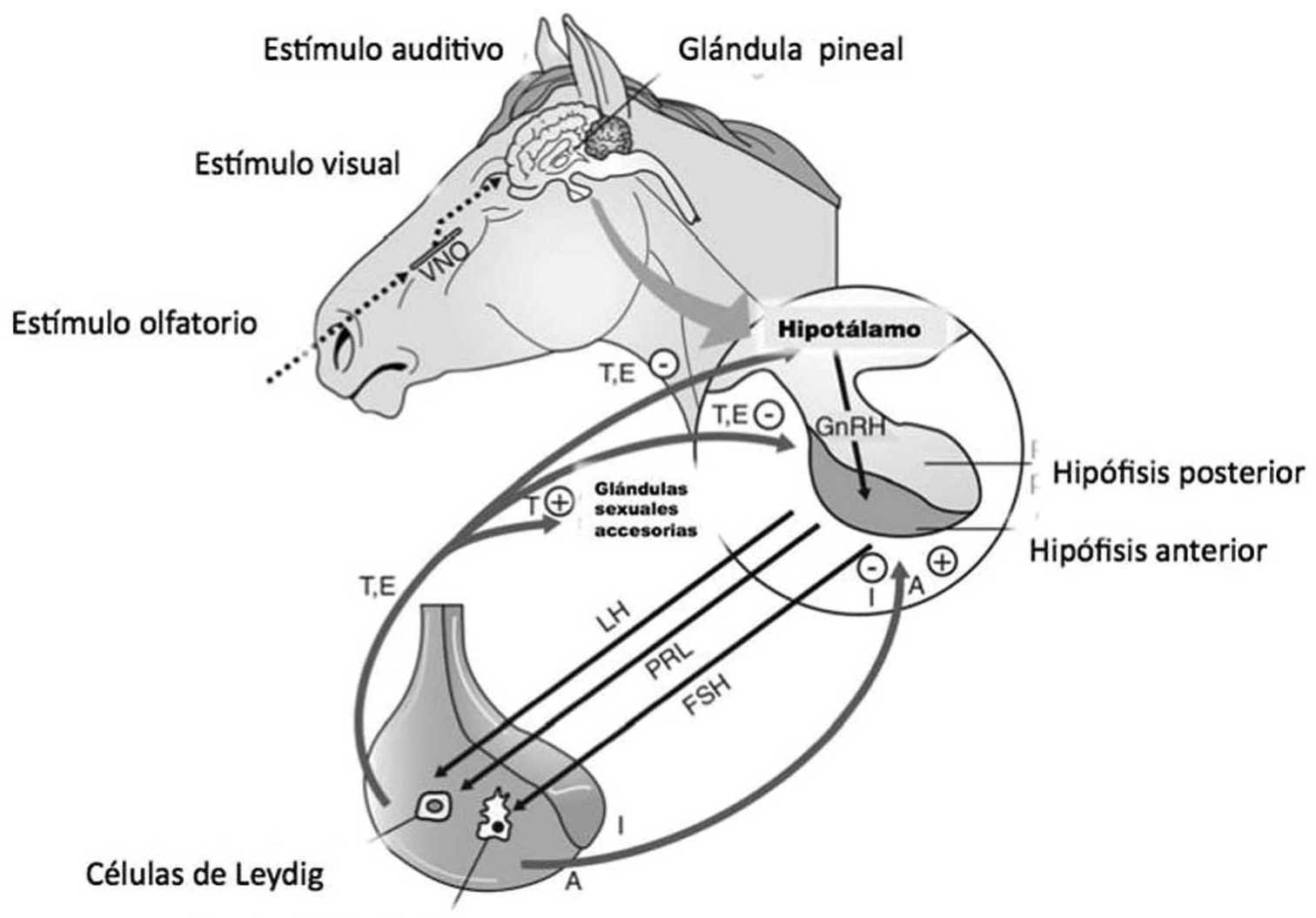

Células de Sertoli

Fig. 3. Retroalimentaciones positiva (+) y Negativa (-) de la producción y liberación hormonal en el potro. VNO, organo Veronasal; GnRH, Hormona Liberadora de Gonadotrofinas; LH, Hormona Luteinizante; Hormona PRL, Prolactina; FSH, Hormona foliculoestimulante; T, Testosterona; E, Estrogenos; I, Inhibina; A, Activina. Se presentan tambien las potenciales influencias externas en el control del eje (modificado de Samper,1999) 


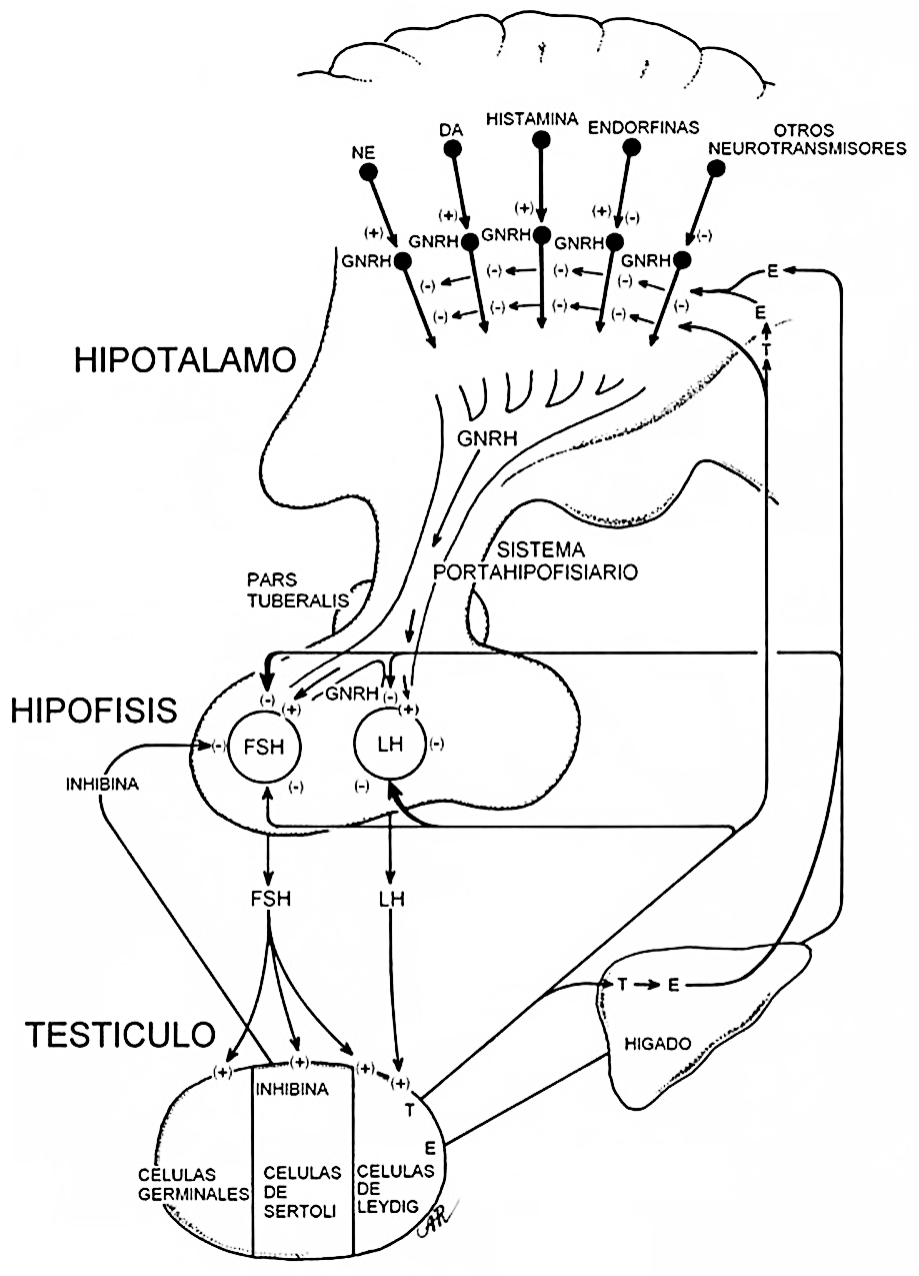

Fig. 4. Intervención de neurotransmisores en la regulación del eje hipotálamo hipófisis testículo (NE: norepinefrina DA: dopamina).

Hormonas liberadoras e inhibitorias. Las neuronas parvocelulares sintetizan hormonas que estimulan o inhiben la liberación de hormonas desde la adenóhipofisis. Estas hormonas se liberan de los terminales nerviosos en la eminencia media y difunden a los capilares del sistema portal hipotálamo hipófisis.

Solo hacia 1970 los factores liberadores hipotalámicos se caracterizaron químicamente. Así la hormona que estimula la liberación de LH también lo hace para FSH $(\mathrm{GnRH})$. Prolactina en cambio obedece a un control inhibitorio del factor inhibidor de prolactina (PIF) o dopamina. Estos factores liberadores son péptidos (decapéptido para $\mathrm{GnRH}$ ).

Transporte de las hormonas liberadoras- inhibitorias. Estas no se acumulan en su sitio de síntesis en los cuerpos neuronales, sino en el sistema magnocelular se transportan rápidamente por el axón para almacenarse en los terminales nerviosos. GnRH ha sido localizada en cuerpos neuronales de la región media basal del hipotálamo (núcleo arcuato) así como en la región anterior (hipotálamo anterior y área pre óptica). GnRH llega al sistema vascular portal de la eminencia media vía axónica o vía líquido cerebro espinal.

Se cree que los tanacitos proyectan a través de la eminencia media a través de estructuras terminales próximas a los vasos portales, descargando allí GnRH. Ambas posibilidades no son excluyentes pero la primera es probablemente la aceptable.

Regulación de las neuronas endocrinas. Existe un control por otras neuronas a través de neurotransmisores sinápticos y por hormonas provenientes del torrente sanguíneo.

\section{NEUROTRANSMISORES (Fig. 4).}

Hay un complejo sistema neuronal que relaciona el hipotálamo con el resto del cerebro, en el cual se incluyen neuronas cuyos cuerpos están fuera del hipotálamo y otras dentro de él. Cualquiera de ellos forma sinapsis con neuronas endocrinas en el cuerpo neuronal o en sus terminales. Una neurona endocrina puede estar en contacto sináptico con varias neuronas clásicas siendo estimulatoria en algún caso e inhibitoria en otro. La actividad por lo tanto en el tiempo resulta de un balance entre señales positivas y negativas. En el proceso reproductivo estas neuronas transmiten información sobre estímulos sensoriales (visual, olfativo). Las neuronas que llegan al hipotálamo y forman sinapsis con neuronas endocrinas facilitan comunicación entre varios núcleos hipotalámicos. En cada sinapsis se descarga un neurotransmisor específico, el que se une a receptores de membrana en la neurona post sináptica, modificando su actividad, en términos de descargas de neurotransmisores u hormonas. Los neurotransmisores han sido difíciles de estudiar respecto a liberación de gonadotrofinas, tres monoaminas intervienen: dopamina y noradrenalina (catecolaminas) y serotonina (indolamina). Se puede obtener cambio en la secreción de LH, FSH y prolactina administrando estas monoaminas o alterando su síntesis o metabolismo.

Dopamina funciona también como hormona ya que es secretada al sistema portal y actúa en la adenohipófisis inhibiendo la secreción de prolactina. Como neurotransmisor probablemente regula la secreción de GnRH. Ello puede explicar que la secreción de gonadotrofinas tiende a bajar cuando sube la secreción de prolactina. 
También han adquirido importancia los péptidos opioides. Uno de ellos (beta endorfina) se encuentra en alta concentración en el hipotálamo y en la sangre del sistema portal de la pituitaria. Los opioides inhiben la secreción de FSH y LH y estimulan la de prolactina, en tanto que sus antagonistas estimulan la secreción de gonadotrofinas. GnRH puede también ser un neurotransmisor débil en el control de la conducta sexual.

Regulación hormonal. Un segundo nivel de control de las neuronas endocrinas lo ejercen por un mecanismo de retroalimentación hormonas del torrente sanguíneo. Los esteroides gonadales se unen a receptores en neuronas tal como lo hacen en otros tejidos blanco para ejercer acción genómica y modificar la síntesis proteica, aunque los efectos rápidos de los esteroides son mediados por otros mecanismos. Muchas preguntas respecto a la interrelación entre todos estos factores aun no se pueden resolver.

Los sitios de unión de esteroides gonadales no solo se encuentran en el hipotálamo sino que se distribuyen ampliamente en el cerebro, lo que sugiere que la acción de estos es muy amplia. Cualquiera sea el caso el cambio en el ritmo de descarga de las hormonas hipotalámicas hacia la circulación portal resulta en una modulación de la secreción de las hormonas hipofisiarias.

Control de la secreción de gonadotrofinas. La secreción de LH y FSH es controlada por dos sistemas de regulación: el sistema tónico y uno cíclico.

El sistema tónico responde por niveles basales permanentes que favorecen el desarrollo de los elementos germinales y endocrinos en la gónada, en cambio el sistema cíclico de secreción responde por un alza masiva en las gonadotrofinas circulantes, como la que causa ovulación en la hembra.

En algunas especies el proceso de diferenciación sexual que ocurre en el cerebro deja a la adenohipófisis sexualmente indiferenciada.

Hay grandes diferencias entre especies respecto a picos de actividad de gonadotrofinas y en algunas ello es estimulado por el acto de la cópula (ovuladores reflejos).

En la mayoría de las especies (ovuladores espontáneos) esto no es así y ocurre por influencias estimulatorias de hormonas gonadales.

Control de la retroalimentación en la secreción de gonadotrofinas. Dentro de límites definidos, cada hormona regula su propia tasa de secreción, como ocurre en el eje hipotálamo hipófisis gónada, que comprende mecanismos de retroalimentación de asa larga, corta y ultracorta. Esta última se refiere a acción neuronal de hormonas hipotalámicas que regulan su propia secreción.

La mayor parte de la retroalimentación es negativa, aunque en algunos casos es positiva cuando la hormona estimula su propia liberación. Los estrógenos, progesterona y andrógenos ejercen una retroalimentación negativa.

El generador de pulsos hipotalámicos. Este es uno de los aspectos más relevantes en la regulación neuroendocrina de la reproducción. En general la secreción tónica de gonadotrofinas, principalmente LH, no ocurre en forma constante, sino en descargas separadas por periodos de baja o nula secreción.

Ello ha llevado a la idea de que la secreción tónica es controlada por un oscilador (generador de pulsos) que reside en el sistema nervioso central, el cual genera descargas de GnRH de las neuronas endocrinas hipotalámicas, ocasionando a su vez pulsos de secreción de gonadotrofinas

El generador de pulsos parece residir en la región medio basal del hipotálamo, en algunas especies en que se le ha podido localizar.

Se ha demostrado en el mono que la naturaleza pulsátil de la secreción de GnRH es necesaria para la secreción gonadotrófica normal y un normal proceso reproductivo.

Control del generador de pulsos. Este es normalmente modificado por varios estímulos que provienen del medio interno y externo. La frecuencia y amplitud de los pulsos de gonadotrofinas normalmente sufren grandes fluctuaciones. Estos cambios son en parte debidos a variaciones de hormonas gonadales que actúan en distintos sitios del eje hipotálamo hipófisis, así la testosterona inhibe en el macho la frecuencia de pulsos de LH. Grandes cambios del generador de pulsos ocurren en la pubertad y también en las distintas estaciones del año para los reproductores estacionales, así como en respuesta a estímulos agudos externos (visual, olfatorio o táctil).

\section{CARACTERISTICAS REPRODUCTIVAS ENANIMA- LES DE REPRODUCCIÓN ESTACIONAL Y CONTI- NUA}

Características reproductivas en el potro (generalidades). El potro es un reproductor estacional cuya estacionalidad es mucho menos marcada que la de la yegua siendo capaz de reproducirse a lo largo de todo el año si existen yeguas en celo. 
La estacionalidad sin embargo afecta el volumen seminal, la concentración espermática en la fracción libre de gel, el recuento total y el número de montas, así como el tiempo de reacción para montar a la yegua (Pickett et al., 1970; 1975, Johnson, 1991) pero la producción espermática es continua a lo largo del año y no está controlada por cambios hormonales cíclicos. El análisis citoquímico del eyaculado de potro muestra diferencias en el núcleo de los espermatozoides en la estación de monta y fuera de ésta, En la época no reproductiva se constata un contenido relativo disminuido de proteínas $\mathrm{SH}$ aunque la proteína total no varía y una menor lectura citofotométrica del DNA. Estos cambios sugieren que el gameto fuera de estación reproductiva es hipermaduro, de modo que, el test de descondensación de la cromatina por el método de Calvin \& Bedford (1971) muestra que el $12 \%$ de los espermatozoides se descondensan luego de una hora de tratamiento, en tanto que los dos tercios se descondensan en época reproductiva. Los espermatozoides fuera de estación reproductiva tienen baja capacidad fecundante y provocan probablemente alta mortalidad embrionaria (Bustos-Obregón, 1980).

Testículo. El testículo se localiza en la bolsa escrotal lo que le permite mantener una temperatura aproximadamente 3 grados inferior a la temperatura corporal $\left(39^{\circ} \mathrm{C}\right)$. La producción espermática es máxima a menor temperatura.

El plexo pampiniforme hace que la arteria testicular se divida en una densa red capilar antes de entrar al testículo. Ella permanece en contacto estrecho con las venas del retorno venoso que también se dividen en un plexo capilar de modo que la sangre que entra al testículo, por lo tanto, pierde calor hacia el retorno venoso.

Las dimensiones del testículo son 6-12 cm de largo con una altura de $4-7 \mathrm{~cm}$ y un ancho de $5-6 \mathrm{~cm}$, respectivamente y puede pesar entre 300 y 350 gramos el par. El tamaño guarda relación con el peso corporal y tiene su talla final en el animal de alrededor de 5 años. El órgano se encuentra dividido en lóbulos, cada uno de los cuales engloba una masa de túbulos seminíferos y áreas intertubulares. En este tejido se lleva a cabo la función espermatogénica así como la función esteroidogénica (endocrinocitos intersticiales intertubulares).

Los sustentocitosque se ubican en los túbulos seminíferos actúan como células de sostén y nutricias para la línea espermatogénica que progresa hacia el lumen hasta la producción final de espermátidas elongadas que se liberan durante la espermiación.

El número de células sustentaculares varía según la estación del año siendo significativamente mayor en la épo- ca reproductiva que corresponde a la mayor producción espermática (Johnson \& Tatum, 1989).

Este incremento en número se corresponde también con un aumento en la longitud de los túbulos seminíferos. No hay constancias de que ocurran cambios similares en el intersticio.

Espermatozoides. La duración total de la espermatogénesis en potro es de alrededor de 57 días y ocurre en ondas sucesivas. La producción espermática promedio diaria del animal maduro sexualmente es del orden de 7 a $8 \times 10^{\wedge} 9$ espermatozoides. El transito epididimario toma entre 8 y 11 días (Dinger \& Noiles, 1986). La producción espermática total está relacionada al volumen testicular el cual puede medirse por medio de Callipers o por ultrasonido.

Al eyacular el potro secreta semen en una serie de hasta 9 chorros cuyo volumen depende de la concentración espermática la cual va decreciendo.

El semen comprende el plasma seminal y los espermatozoides y tiene un aspecto lechoso y consistencia gelatinosa fluida. La concentración espermática varía según la fracción que se considere. La fracción preespermática o inicial no contiene espermatozoides. Cumple una función lubricante de la uretra antes de la eyaculación.

La fracción rica en espermatozoides se secreta tan pronto el glande aumenta de volumen para forzar su entrada en el Cervix, tiene un volumen de 40 a $80 \mathrm{ml}$ y contiene $80-90 \%$ de los espermatozoides y de los componentes bioquímicos del semen. La tercera fracción o fracción gelificada tiene un volumen muy variable que alcanza hasta $80 \mathrm{ml}$ y depende del grado de libido del animal. Esta fracción es modificada por la estación, siendo más baja en el periodo no reproductivo (Squires et al., 1979).

Su volumen es alterado por la edad así como también el recuento espermático.

Cambios fisiológicos. En el control endocrino de la reproducción del potro interviene el eje hipotálamo hipófisis gónada. Los estímulos ambientales tales como, el largo del día y la temperatura tienen un efecto importante sobre este eje. La estacionalidad es controlada por la secreción de melatonina por la glándula pineal en respuesta a la longitud del día. Tanto melatonina como el fotoperíodo pueden en la hembra controlar la cronología de la estación reproductiva pero la sobre estimulación con días largos artificiales prolongada lleva a refractariedad (Cox et al., 1988; Argo et al., 1991). 
Las concentraciones de prolactina en el macho también son influidas por la longitud del día. Cuando ésta aumenta sube la secreción de prolactina. Se cree que ésta también controla el crecimiento y la ganancia de peso del animal (Tucker \& Wetterman, 1976).

LH y FSH. Ambas son secretadas por el estímulo de la GnRH hipotalámica. Las concentraciones plasmáticas de LH y FSH en el potro sexualmente activo son del orden de 3-4 $\mathrm{ng} / \mathrm{ml}$ y $7-7.5 \mathrm{ng} / \mathrm{ml}$ respectivamente (Amann 1993; Kainer, 1993).

Testosterona. Los testículos contienen células somáticas que son el blanco de LH y FSH. La secreción de testosterona es controlada por LH (Amann, 1981) y se produce de manera pulsátil por lo cual la toma de muestra sanguínea debe ocurrir al tiempo de máxima secreción para tener el valor real de testosterona del animal.

Tanto FSH como testosterona influyen en la fisiología de la célula de Sertoli y ambas son responsables de iniciar el proceso de espermatogénesis favoreciendo el desarrollo de las espermatogonias hasta la producción de espermatocitos secundarios. La testosterona completa este desarrollo hasta el espermatozoide que se libera para ser conducido al epidídimo donde experimentará el proceso de maduración. La testosterona además controla el desarrollo de los genitales masculinos y del descenso testicular. En el feto o el neonato, los cambios puberales y el crecimiento acelerado del animal y la función de las glándulas anexas al tracto masculino. También es responsable de la libido y de la conducta sexual, así como del desarrollo muscular. La testosterona ejerce retroalimentación negativa sobre la adenohipófisis reduciendo la liberación de LH y FSH de parte de ésta. Un derivado de la testosterona (DHT) también ejerce este efecto negativo aunque más débilmente. Hay una pequeña contribución de producción de testosterona por parte de la suprarrenal.

Los andrógenos tienen además un ritmo de secreción diurna y en el potro se ha reportado que están elevados a las 6 A.M. y a las 18:00 horas, rasgo adaptativo que permite la cópula en horas de menor peligro de que los animales sean víctimas de sus predadores (Pickett et al., 1989).

Inhibina y activina. Ambas producidas por los sustentocitosse relacionan con la producción espermática total y tienen un efecto adicional de retroalimentación sobre el hipotálamo y la hipófisis respecto a la producción de FSH (retroalimentación negativa y positiva respectivamente) pero su rol especifico en el potro no se conoce (Roser et al., 1997).

Prolactina. Se supone que su principal rol es el de transductor del número de horas luz diarias a cambios fisiológicos reproductivos estacionales y al efecto de LH estimulatorio sobre las endocrinocitos intersticiales y de esta manera también controlando el funcionamiento de las glándulas anexas del tracto genital (Thompson et al.,1996), aunque estas funciones no se han descrito específicamente para el potro.

Estrógenos. El testículo de potro contiene una alta concentración de estrógenos y estrona $(150-200 \mathrm{pg} / \mathrm{ml})$ y es la más alta en el testículo de mamíferos. Su significado es aún desconocido.

Cambios conductuales. La testosterona controla la conducta del macho en relación con la reproducción aunque hay gran variación individual, probablemente de causa genética.

A la vista de una hembra en celo la frecuencia y amplitud de GnRH, y en consecuencia de la liberación de LH y FSH aumenta, aumentando la producción de testosterona y la conducta copulatoria del macho. Un $20 \%$ de la GnRH liberada actúa directamente sobre centros cerebrales para modificar la conducta (Pozor et al., 1991). El macho fija sus ojos en la hembra, arquea el cuello, eleva el labio superior y a menudo tiene lordosis de la columna y emite un relincho particular. Si la hembra es receptiva el macho se acercará en un cortejo que ha sido descrito detalladamente hasta posicionarse detrás de la hembra, generalmente en el lado izquierdo, e iniciar algunos intentos de monta para observar la reactividad de la hembra. Si ésta es receptiva la eyaculación sobreviene muy pronto después de la penetración y se acompaña de movimientos rítmicos de la cola. Todos estos eventos están controlados por el nivel de testosterona, y al término de la estación reproductiva el tipo de reacción del macho se prolonga a medida que declinan los niveles de testosterona y su libido también disminuye (Weber \& Woods, 1993).

TORO. El toro en cuanto a reproducción puede ser usado en monta natural o como donante de semen para inseminación artificial. En el primer caso servirá hasta alrededor de 60 o más vacas, y en el segundo caso a cientos de vacas receptoras. El semen se puede obtener por eyaculación tanto en la vagina natural como artificial.

La pubertad en el toro se define como aquel animal que produce espermatozoides suficientes para impregnar exitosamente a la hembra o en términos prácticos, como el que produce un eyaculado de al menos 50 millones de espermatozoides con un mínimo de $10 \%$ móviles. La pubertad se asocia a un periodo de rápido crecimiento testicular con un cambio del patrón de liberación de LH y un alza de la testosterona plasmática a la vez que se inicia la espermatogénesis completa. Los estadios de desarrollo testicular han sido definidos por Fossland \& Schultz (1961). 
El estadio 4 de desarrollo ocurre entre los 7 y 9 meses de edad en que puede coincidir la primera cópula del animal con el desarrollo de una capacidad fecundante o incluso ser anterior a este momento. El volumen de semen, el número total de espermatozoides y el de espermatozoides motiles van aumentando en los meses subsiguientes así como la fertilidad definida como el no retorno a estro de las vacas inseminadas.

Función testicular. La tasa de secreción de LH aumenta en las primeras semanas de desarrollo postnatal alcanzando alta concentración entre 2 a 5 meses de edad. Tanto LH como testosterona se secretan en pulsos de amplitud y frecuencia crecientes. A medida que aumenta la testosterona aumenta también su efecto de retroalimentación negativa sobre LH . Las concentraciones plasmáticas de FSH en cambio cambian muy poco durante la prepubertad, época en la que también se secretan en forma pulsátil y sincrónica con LH.

La testosterona se relaciona con la producción espermática a través de su acción sobre las células sustentaculares. La espermatogénesis completa en el toro toma aproximadamente 60 días. La concentración adecuada de espermatozoides requiere de testosterona y la secreción de FSH es regulada por la secreción de inhibina efectuada por la célula de Sertoli.

Transporte espermático en el toro. El transporte en el epidídimo de los espermatozoides formados en el testículo hace que éstos progresen hasta el conducto deferente proximal. Normalmente $30-35 \%$ de los espermatozoides se encuentran en la cabeza y cuerpo, $50-55 \%$ en la cola y $10-$ $15 \%$ en la ampolla del deferente.

La frecuencia del eyaculado influencia el tiempo de tránsito en la cola pero no en los otros segmentos. El tiempo promedio de tránsito en cabeza y cuerpo es de 2 a 3 días y en cola de 3 a 5 días.

Producción espermática en el toro. La frecuencia de eyaculación dentro de ciertos límites no afecta la capacidad fecundante del semen, ya que no hace variar significativamente el tránsito epididimario. El número de espermatozoides en un animal que no ha eyaculado por 7 a 10 días es máximo y puede reducirse en un $25 \%$ con eyaculación día por medio. La cantidad de espermatozoides producida en el animal adulto normal varia con la raza y se correlaciona con el tamaño testicular.

Composición del semen. El volumen del semen en cada eyaculado varía entre distintos animales y puede ser de 1$2 \mathrm{ml}$ en el animal joven hasta 15 en animales mayores, siendo en promedio de 5 a $6 \mathrm{ml}$ por eyaculado. La concentra- ción espermática puede variar desde 0 (azoospermia) hasta $300 \times 10^{\wedge} 6$ en reproductores excepcionales.

Calidad espermática. Se relaciona principalmente con volumen, concentración, motilidad, proporción de espermatozoides vivos, de anormales, con algunos parámetros bioquímicos y funcionales, siendo muy variable de un animal a otro.

Morfología espermática. Blom (1983) clasificó los defectos de los espermatozoides y propuso un modelo de espermiograma; el examen morfológico del semen bovino se acepta como una técnica importante para diferenciar animales de alta o baja capacidad fecundante. Williams \& Savage $(1925 ; 1927)$ hicieron observaciones importantes de destacar:

a. Las dimensiones de la cabeza del espermatozoide de toros de buena fertilidad muestran escasa variación.

b. No hay toros de alta fertilidad que tengan más de un $17 \%$ de formas anómalas

c. Un número aceptable de formas anómalas en un eyaculado depende del tipo de anormalidades de que se trate.

Los espermatozoides anormales son el resultado de defectos de la espermatogénesis, los cuales pueden ser de origen genético, debido a enfermedades o a condiciones ambientales adversas. Entre éstas destaca la alta temperatura ambiental que aumenta el número de espermatozoides anómalos.

Fertilidad y calidad seminal también son variables con la estacionalidad. El test hiposmótico o el test de penetración en moco cervical, así como los test de fecundación in vitro son los ensayos funcionales más utilizados en bovinos.

Conducta copulatoria en el toro. Cuando la hembra se aproxima al estro se hace atractiva para el toro el cual lo detecta por estímulos olfatorios provenientes de probables feromonas. El macho se aproxima y olfatea el área perineal y después de algunos intentos de monta copula por un tiempo muy corto (algunos segundos), insertando el pene y depositando el semen en la vagina anterior.

Líbido. La libido tiene un importante componente genético probablemente correlacionado con patrones hormonales como la relación estrógeno / testosterona que tiende a ser alta en los toros de baja libido.

También la conducta sexual y libido del animal varia sugiriendo que puede ser influida por factores ambientales o el aprendizaje. 
BUSTOS OBREGÓN, E. \& TORRES, L.Seasonal Reproduction in the male Int. J. Morphol., 30(4):1266-1279, 2012.

SUMMARY: There is a surprising interrelationship between environments and adaptation of reproductive behaviour, very evident in seasonal breeders; which may reproduce in long or short days, according to proximal factors, mainly the light photoperiod which triggers photoneuroendocrine changes. These involve photoreceptors, a clock and the neuroendocrine apparatus. Gonadotropins (GT), gonadal development, negative feed back of GT done by sexual steroid, the intervention of the retino-hypothalamic fibers, and suprachiasmatics nucleus as well as melatonine secretion, intervene in this regulation. Of importance is the pulse generator of the hypothalamus (medial eminence) and its control of adenohypofisis for the secretion of LH and FSH. In the testis interstitial endocrine cells (Leydig)(secreting testosterone and also estrogens), establish a feed back loop with the adenohypofisis and hypothalamus in a circuit of long, short and ultra short circuit with neuroendocrine neurons playing a key role. Sustentocyte intratubular (Sertoli)are also important for their trophic, mechanic and metabolic relationships with the germ cells, and the secretions of activine and inhibine, which triggers or inhibits FSH secretions respectively. Sustentocyte also secrete many specifics proteins among which ABP (Androgen Binding Protein) is important because it concentrates 100 fold testosterone in the testicular parenchyme. Tonic secretion by pulses of GT, mainly LH, is due to hypothalamic activity with the control generation of these pulses by puberty. Reproduction in the stallion and the bull are presented as examples.

KEY WORDS: Seasonal breeding; Hypotalamus-Hypophysis-Testes axis; Neuroendocrinology and reproduction; Reproductive features in stallion and bull.

\section{REFERENCIAS BIBLIOGRÁFICAS}

Abecia, J. A.; Valares, J. A.; Forcada, F.; Palacín, S.; Martín, A. \& Martino, H. The effect of melatonin on the reproductive performance of three sheep breeds in Spain. Small Rumin. Res., 69:106, 2007.

Amann, R. R. A review of the anatomy and physiology of the stallion. Equine Veterinary Science, 1(3): 83-105, 1981.

Amann, R. P. Physiology and endocrinology. In: McKinnon, A. O. \& Voss, J. L. (eds.) Equine Reproduction. Philadelphia, Lea and Febiger, 1993. pp. 658-85.

Argo, C. M.; Cox, J. E. \& Gray, J. L. Effect of oral melatonin treatment on the seasonal physiology of pony stallions. J. Reprod. Fertil., 44:115-25, 1991

Berndtson, W. E.; Squires, E. L. \& Thompson, D. L. Jr. Spermatogenesis, testicular composition and the concentration of testosterone in the equine testis as influenced by season. Theriogenology, 20(4):449-57, 1983.

Bustos-Obregón, E. Cytochemical characterization of sperm nucleus during epididymal maturation in mammals. Arch. Biol. Med. Exp., 13:335-41, 1980 .

Blom, E. Pathological conditions in the genital organs and in the semen as ground for rejection of breeding bulls for import or export to and from Denmark. Nordic Vet., 35:105-30, 1983.

Calvin, H. \& Bedford, J. M. Formation of disulphide bonds in the nucleus and accesory structures of mammalian spermatozoa during maturation in the epididymus. J. Reprod. Fertil. Suppl., (suppl. 13):65-75, 1971.

Carter, D. S. \& Goldman, B. D. Antigonadal effects of timed melatonin infusion in pinealectomized male Djungarian hamsters (Phodopus sungorus sungorus): duration is the critical parameter. Endocrinology, 113:1261-7, 1983.
Chemineau, P.; Guillaume, D.; Migaud, M.; Thiery, J. C.; PellicerRubio, M. T. \& Malpaux, B. Seasonality of reproduction in mammals: intimate mechanisms and practical applications. Reprod. Domest. Anim., 43(suppl 2):40-47, 2008.

Coelho, L. A.; Rodrigues, P. A.; Nonaka, K. O.; Sasa, A.; Balieiro, J. C.; Vincente, W. R. \& Cipolla-Neto, J. Annual pattern of plasma melatonin and progesterone concentrations in hair and wool ewe lambs kept under natural photoperiod at lower latitudes in the southern hemisphere. J. Pineal Res., 41:101-7, 2006.

Courot, M. \& Ortavant, R. Endocrine control of spermatogenesis in the ram. J. Reprod. Fertil. Suppl., 30:47-60, 1981.

Cox, J. E.; Redhead, P. H. \& Jawad, N. N. The effect of artificial photoperiod at the end of the breeding season on plasma testosterone concentrations in stallions. Aust. Vet. J., 65:239-41, 1988.

Dinger JE, Noiles EE. Prediction of daily sperm output in stallions. Theriogenology, 26(1):61-7, 1986

Duncan, M. J. Circannual prolactin rhythms: calendar-like timer revealed in the pituitary gland. Trends Endocrinol. Metab., 18:259-60, 2007.

Elliott, J. A. Circadian rhythms and photoperiodic time measurement in mammals. Fed. Proc., 35:2339-46, 1976.

Fossland, R. G. \& Schultz, A. B. A histological study of the postnatal development of the bovine testis. University of Nebraska Agriculture Experimental Station Research Bulletin. 199:3-16 1961.

Hattar, S.; Liao, H. W.; Takao, M.; Berson, D. M. \& Yau, K. W. Melanopsin containing ganglion cells: architecture, projections, and intrinsic photosensitivity. Science, 295:1065-70, 2002.

Haresign W. Manipulation of reproduction in sheep. J. Reprod. Fertil. Suppl., 45:127-139, 1992. 
Johnson, L. \& Thompson D. L. Jr. Age-related and seasonal variation in the Sertoli cell population, daily sperm production and serum concentrations of follicle-stimulating hormone, luteinizing hormone and testosterone in stallions. Biol. Reprod., 29:777-89, 1983.

Johnson, L. Seasonal differences in equine spermatocytogenesis. Biol. Reprod., 44:284-91, 1991.

Johnson, L, \& Tatum, M. E. Temporal appearance of seasonal changes in numbers of Sertoli cells, Leydig cells, and germ cells in stallions. Biol. Reprod., 40:994-9,1989.

Kainer, R. A. Reproductive organs of the mare. In: Equine Reproduction. McKinnon, A. O. \& Voss, J. L. (eds.) Philadelphia, Lea and Febiger, 1993. pp. 3-19.

Kappers, J. A. The mammalian pineal gland, a survey. Acta Neurochir, 34:109-49, 1976.

Moore, R. Y.; Speh, J. C. \& Card, J. P. The retino-hypothalamic tract originates from a distinct subset of retinal ganglion cells. J. Comp. Neurol., 352:351-66, 1995.

Morgan, P. J. \& Hazlerigg, D. G. Photoperiodic signaling through the melatonin receptor turns full circle. J. Neuroendocrinol., 20:820 6, 2008.

Panda, S.; Nayak, S. K.; Compo, B.; Walker, J. R.; Hogenesch, J. B. \& Jegla, T. Illumination of the melanopsin signaling pathway. Science, 307:600-4, 2005.

Pickett, B. W.; Faulkner, L. C. \& Sutherland, T. M. Effect of month and stallion on seminal characteristics and sexual behaviour. $J$. Anim. Sci., 31:713-28, 1970.

Pickett, B. W.; Faulkner, L. C. \& Voss, J. L. Effect of season on some characteristics of stallion semen. J. Reprod. Fertil. Suppl., 23:258,1975 .

Pickett, B. W.; Amann, R. P.; McKinnon, A. O.; Squires, E.L. \& Voss, J. L. Management of the stallion for maximum reproductive efficiency. II. In: Bulletin of the Colorado State University Agriculture Experimental Station Animal Reproduction Laboratory General Series Bulletin No. 05. Colorado State University, Fort Collins, 1989. pp. 121-5.

Pozor, M., McDonnell, S.M., Kenney, R.M. \& Tischner, M. GnRH Facilitates Copulatory Behaviour in Testosterone-treated Geldings. J. Reprod. Fert. Suppl., 44:666-7, 1991.

Reppert, S. M. Melatonin receptors: molecular biology of a new family of G protein-coupled receptors. J. Biol. Rhythms, 12:528-31, 1997.

Revel, F. G.; Ansel, L.; Klosen, P.; Saboureau, M.; Pévet, P.; Mikkelson, J. D. \& Simonneaux, V. Kisspeptin: a key link to seasonal breeding. Rev. Endocrinol. Metab. Disord., 8:5765, 2007.

Reiter, R. J. \& Hester, R. J. Interrelationships of the pineal gland, the superior cervical ganglia and the photoperiod in the regulation of the endocrine systems of hamsters. Endocrinology, 79:1168-70, 1966.
Reiter, R. J. Pineal control of a seasonal reproductive rhythm in male golden hamsters exposed to natural daylight and temperature. Endocrinology, 92:423-30, 1973.

Reiter, R. J. The pineal and its hormones in the control of reproduction in mammals. Endocr. Rev., 1:109-31, 1980.

Reiter, R. J. Melatonin: the chemical expression of darkness. Mol. Cell Endocrinol., 79:C153-8, 1991.

Reiter, R. J. The melatonin rhythm: both a clock and a calendar. Experientia, 49:654-64, 1993.

Reiter, R. J.; Tan, D. X.; Manchester, L. C.; Paredes, S. D.; Mayo, J. C. \& Sainz, R. M. Melatonin and reproduction revisited. Biol. Reprod., 81:445-56, 2009.

Roser, J. F. Endocrine basis for testicular function in the stallion. Theriogenology, 48(5):883-92, 1997.

Samper, J. C. Equine Breeding Management and Artificial Insemination. $2^{\circ}$ ed. Saunders, 1999.

Squires, E. L., Pickett, B. W. \& Amann, R. P. Effect of successive ejaculation on stallion seminal characteristics. J. Reprod. Fertil. Suppl., 27:7-12, 1979.

Thompson, C. H.; Thompson, D. L. Jr.; Kincaid, L. A. \& Nadal, M. R. Prolactin involvement with increase in seminal volume after sexual stimulation in stallions. J. Anim. Sci., 74(10):2468-72, 1996.

Tucker, H. A. \& Wetterman, R. P. Effects of ambient temperature and relative humidity on serum prolactin and growth hormone in heifers. Proc. Soc. Exp. Biol. Med., 151:623-6, 1976.

Wagner, G. C.; Johnston, J. D.; Clarke, I. J.; Lincoln, G. A. \& Hazlerigg, D. G. Redefining the limits of day length responsiveness in a seasonal mammal. Endocrinology, 149:32-9, 2008.

Weber, J. A. \& Woods, G. L. Ultrasonic measurements of stallion accessory glands and excurrent ducts during seminal emission and ejaculation. Biol. Reprod., 49(2):267-73, 1993.

Williams, W. W. \& Savage, A. W. L. Observations on the seminal micropathology of bulls .Cornell Vet., 15:353-75, 1925.

Williams, W. W. \& Savage, A. W. L. Methods of determining the reproductive health and fertility of bulls. A review with additional notes. Cornell Vet., 17:374-6, 1927.

Young K. A. \& Nelson, R. J. Mediation of seasonal testicular regression by apoptosis. Reproduction. 122:677-85, 2001.

Dirección para correspondencia:

Eduardo Bustos-Obregón

Laboratorio de Biología de la Reprodución, ICBM,

Facultad de Medicina, Universidad de Chile,

Santiago

CHILE

Recibido : 28-02-2012

Email:ebustos@med.uchile.cl
Aceptado: 14-07-2012 